\title{
MONOCLONAL GAMMOPATHY IN A PATIENT WITH RELAPSING POLYCHONDRITIS: CASE REPORT OF AGGRESSIVE EVOLUTION
}

Andre Silva Franco ${ }^{1}$, Isabele Parente de Brito Antonelli ${ }^{1}$, Henrique Ayres Mayrink Giardini ${ }^{1}$, Lissiane Karine Noronha Guedes ${ }^{1}$, Rosa Maria Rodrigues Pereira1,*

1. Universidade de São Paulo, São Paulo (SP), Brazil.

*Corresponding author: rosamariarp@yahoo.com

\section{BACKGROUND}

Monoclonal gammopathy is frequently associated with rheumatic diseases and has variable clinical significance - which includes monoclonal gammopathy of undetermined significance (MGUS), multiple myeloma, plasmacytoma, Waldenström's macroglobulinemia, lymphoma, monoclonal gammopathy of renal significance, primary amyloidosis, among others. Systemic involvement of multiple organs is well described in several of these entities and monoclonal gammopathy is often associated with rheumatic diseases.

\section{CASE REPORT}

S.R.A.C., 58-year-old female, in outpatient follow-up due to relapsing polychondritis since 1988 , with nasal and auricular chondritis and sensorineural hearing loss, without immunosuppression for 15 years. She also had chronic kidney disease in peritoneal dialysis (multifactorial etiology due to arterial hypertension and nonsteroidal anti-inflammatory overuse) for 8 years. In August2019, a monoclonal gammopathy was identified $(0.4 \mathrm{~g} / \mathrm{dL}$ monoclonal peak in serum protein electrophoresis) - hematology evaluation suggested regular clinical assessment for hypothesis of low-risk MGUS. After 9 months, she was hospitalized to investigate neuropathic pain in lower limbs lasting 4 months, with electroneuromyography confirming length-dependent symmetrical sensorimotor polyneuropathy with axonal predominance. In addition, she presented with an intrahepatic cholestatic pattern of liver injury, alternating periods of constipation and diarrhea, and dysautonomia with significant postural hypotension and tachycardia at rest. Complementary tests showed stable monoclonal peak with kappa/lambda free light chain ratio of 0.34 and echocardiography revealed increased myocardial thickness. Histologic evaluation of abdominal fat pad aspirate and bone marrow biopsy were negative for amyloid deposition. Due to high suspicion, a second bone marrow biopsy was warranted, with positive result for amyloid protein. Further evaluation found $6 \%$ of CD138 positive plasma cells with predominance of lambda light chains, suggesting primary amyloidosis due to lambda chain secreting plasma cell neoplasm, leading to cardiac, hepatic and neurological dysfunction. The patient presented unfavorable evolution, with progressive dysautonomia, culminating in circulatory collapse and death.

\section{CONCLUSION}

The reported case highlights relevant points for the rheumatologist regarding monoclonal gammopathy: The high prevalence in rheumatic diseases, and the association with several conditions, including primary amyloidosis, which presents with multisystemic impairment (heart failure with preserved ejection fraction, hepatosplenomegaly with intrahepatic cholestasis, peripheral neuropathy, cardiovascular and gastrointestinal dysautonomia, renal dysfunction and proteinuria). It is difficult to diagnose in early stages of the disease and requires high suspicion for the correct diagnosis and management. 